\title{
Coarctation of Aorta
}

\section{Naveen Sheikh* \\ Dipal Krishna Adhikary}

Department of Cardiology Bangabandhu Sheikh Mujib Medical University Dhaka, Bangladesh.
*Correspondence to:

\section{Dr. Naveen Sheikh}

Assistant Professor

Department of Cardiology

Bangabandhu Sheikh Mujib Medical University

Dhaka, Bangladesh.

Mobile : 01711934490

E-mail: sheikhnaveen@yahoo.com.sg

\section{INTRODUCTION}

Coarctation derived from Latin term coartatio, which translated literally means a drawing together. When used in the context of congenital heart disease, Coarctation refers to an area of narrowing of the thoracic aorta in the region of the insertion of arterial duct, with or without additional abnormalities of the aortic $\operatorname{arch}^{1}$.

First description of aortic Coarctation is generally attributed to Johann Freidrich Meckel the famous Prussian anatomist who presented an 18 year old female to Royal Academy of Sciences of Berlin in 1750.

\section{Prevalence and Etiology}

Aortic Coarctation accounts for $6-8 \%$ life births with congenital heart disease. As with most left-sided obstructive lesions, coarctation occurs more commonly in males than in females, with a male: female ratio ranging from 1.27 to 1.74 . The genetic component to coarctation has long been recognized in the Turner XO syndrome, in which about $35 \%$ of patients are affected. Environmental influence on the development of coarctation also has been suggested by a study detecting a seasonal variation, with the incidence of coarctation peaking in the late fall and winter.

\section{Pathology}

Aortic Coarctation cannot, however be considered to be a uniform entity. Instead, it represent spectrum of lesions, generally encompassing variable degrees of tubular hypoplasia along with additionally stenotic areas within aortic arch (neonates \& infants) to more discrete coarctation of the upper thoracic aorta in association with arterial collateral \& degenerative changes (later age).

Less commonly, coarctation of the aorta occurs in other locations, such as the ascending aorta or the abdominal aorta. Coarctation of the abdominal aorta is a complex lesion that often is associated with renal artery stenosis.

\section{Histologic Examination}

Histologic examination of the coarcted aortic segment discloses thick intimal and medial ridges that protrude posteriorly and laterally into the aortic lumen .The ductus or ligamentum arteriosus inserts at the same level anteromedially. Intimal proliferation and disruption of elastic tissue may occur distal to the coarctation (the jet lesion) at a site where high-velocity flow impacts the arterial wall. It is this distal site where infective endarteritis, intimal dissections, or aneurysms may occur ${ }^{2}$.

\section{Associated Anomalies}

Intracardiac pathology commonly is associated with coarctation of the aorta ${ }^{3}$. Simple coarctation describes coarctation of the aorta occurring without important intracardiac lesions, with or without a patent ductus arteriosus. The term complex coarctation is used to describe coarctation with important associated intracardiac pathology. Children who present in infancy are much more likely than older children to have complex Coarctation. A large ventricular septal defect is the most common associated lesion. A bicuspid aortic valve occurs upto $85 \%$ of patients with coarctation, and the valve may be stenotic or the annulus hypoplastic. Mitral stenosis also occurs in patients with coarctation and may be caused by a supravalvar mitral ring or the presence of a single parachute papillary muscle (13). 
The association of multiple left-sided obstructive lesions with coarctation has been referred to as Shone syndrome (14) and constitutes a challenging group of lesions when treatment is required in infancy. Extracardiac vascular anomalies are present in many patients with coarctation. The most important of these anomalies include variations in the brachiocephalic artery anatomy, a collateral arterial circulation, and berry aneurysms of the circle of Willis ${ }^{4}$.

\section{Morphogenesis}

Three main aberrations in embryological development have been proposed to explain abnormalities of the aortic arch.

$>0$ Abnormal embryogenesis of the vessels of the arch-

$>0$ Abnormal development of the arterial duct (ductus tissue theory)- Coarctation develops as the result of migration of ductus smooth muscle cells into the periductal aorta, with subsequent constriction and narrowing of the aortic lumen

$>0$ Changes in the ratio of flow between pulmonary and systemic arterial pathways (hemodynamic theory) Coarctation results from reduced volume of blood flow through the fetal aortic arch and isthmus.

\section{Hemodynamics}

Various compensatory mechanisms assist the left ventricle in response to the increase in outflow impedance. Left ventricular myocardial hypertrophy is perhaps the most important. Myocardial hypertrophy tends to normalize myocardial wall stress and ventricular afterload.

If the coarctation is severe or develops rapidly, as in a newborn on ductal closure, left ventricular systolic dysfunction and congestive heart failure may ensue. The hemodynamic consequences include diminished stroke volume, increased left ventricular end-diastolic pressure, elevated left atrial pressure, pulmonary venous congestion, and pulmonary artery hypertension Systolic arterial hypertension is a manifestation of the aortic constriction, but it probably also reflects changes in vascular reactivity, arterial wall compliance, and baroreceptor reflex function ${ }^{5-7}$.

\section{Clinical Features}

The clinical presentation of coarctation of the aorta generally follows one of three patterns: An infant with congestive heart failure, a child or adolescent with systemic arterial hypertension, or a child with a heart murmur. When coarctation presents in infancy, it often presents as a catastrophic illness. Congestive heart failure and shock may occur suddenly as the ductus arteriosus closes.

Coarctation of the aorta often presents later in childhood as systolic hypertension or as a heart murmur. Delayed diagnosis beyond infancy is common because the physical findings may be subtle and most of these children are asymptomatic. On careful investigation, some children will report lowerextremity claudication with exercise or frequent headaches.

\section{Physical Examination}

In an infant with congestive heart failure, one encounters a pale, irritable child in respiratory distress. Tachycardia, dyspnea, diaphoresis, hepatomegaly, and, rarely, extremity edema may signal the presence of congestive heart failure and low cardiac output. Differential cyanosis may be observed (cyanosis confined to the lower extremities) if a right-to-left ductal shunt is present. In contrast, the appearance of an older child with coarctation may be entirely benign. The hallmark physical findings in coarctation consist of discrepant arterial pulses and systolic blood pressures in the upper and lower extremities. Several murmurs may be present, depending on the nature of the coarctation, associated intracardiac lesions, and the arterial collateral system.

\section{Radiograph}

In infant moderate to severe cardiomegaly is evident, and the pulmonary vascular markings are increased and pulmonary venous congestion may be present.

In older children and adolescents have normal or only mildly enlarged heart size. A localized indentation of the aorta at the site of coarctation ( 3 sign) is common. Rib notching found in older patients but is uncommon in those younger than 5 years of age. It is caused by erosion of the inferior surfaces of posterior ribs by dilated and tortuous intercostal arteries.

\section{Echocardiography}

Two-dimensional echocardiography and Doppler studies provide an accurate, noninvasive assessment of coarctation anatomy and physiology in most patients. Color flow Doppler assists in localizing the site of obstruction and is particularly helpful in cases where two-dimensional imaging is difficult or inconclusive.

\section{Management}

The timing of coarctation repair depends on the nature of the patient's presentation.

\section{Presentation in Infancy}

Medical management consists of initially stabilizing the patient with ionotropic support and diuretic therapy. A critically ill newborn also may benefit from prostaglandin E1 to promote ductal patency and improve perfusion of the descending aorta and renal and mesenteric beds. Metabolic disturbances, such as acidosis, hypothermia, hypoglycemia, or anemia, must be recognized and treated promptly. After a brief period of medical management to stabilize the child, definitive repair should be performed ${ }^{8}$.

Presentation in Childhood

Coarctation repair is generally recommended at 2 to 3 years of age in asymptomatic children without severe upper-extremity hypertension, the risk for late recurrence of coarctation appears to be increased when repair is performed on a patient younger than 1 year of age, because of an increased risk for residual hypertension and early atherosclerotic cardiovascular disease (50), elective repair should not be delayed into late childhood and adolescence.

Surgical repair remains the conventional treatment for most children with coarctation of the aorta. Percutaneous balloon angioplasty is a less-invasive alternative to surgical repair for patients with a discrete coarctation of the aorta ${ }^{9}$. Balloon angioplasty has gained wide acceptance as effective therapy for a recurrent postoperative coarctation but remains somewhat controversial as a primary treatment strategy for a native coarctation. A stent implanted concurrently with 
balloon angioplasty, in either native or postoperative recurrent coarctation, functions as an endovascular buttress to provide support to the dilated aortic segment ${ }^{10}$. Stents decrease coarctation restenosis related to vessel recoil and may also diminish the late incidence of aneurysm formation.

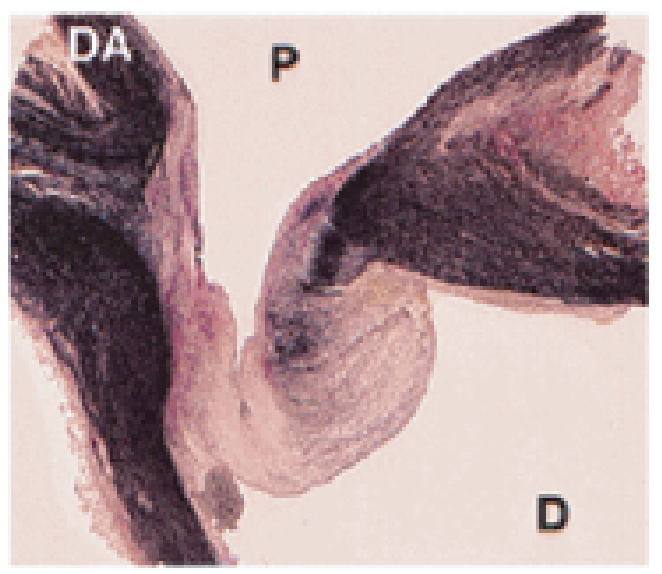

Figure 1: Elastic stain demonstrating disorganized media and intimal proliferation on the diserete posterior shelf on the right, opposite the DA. P, proximal aortic lumen; D, distal aortic lumen

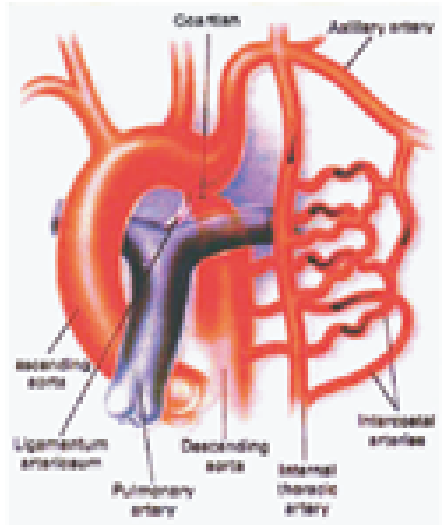

Figure 2: Collateral arterial circulation

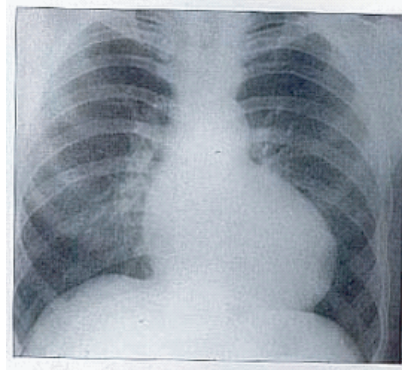

Figure 3: sign

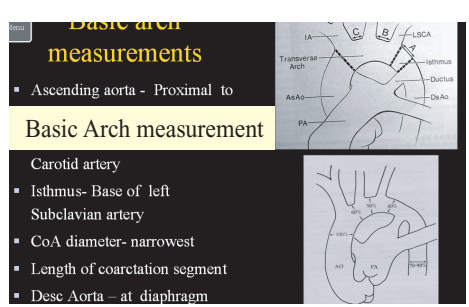

Figure 5: Basic Arch measurement

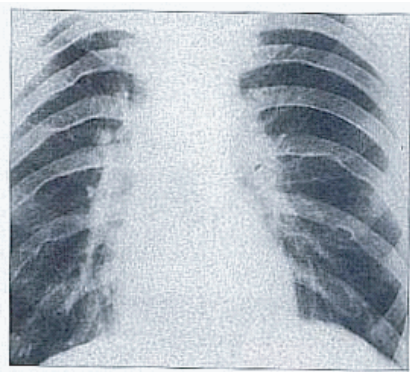

Figure 4: Rib notching

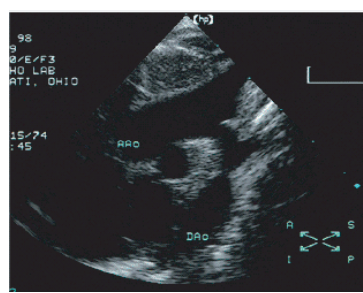

Figure 6: 2D Echo of Coarctation
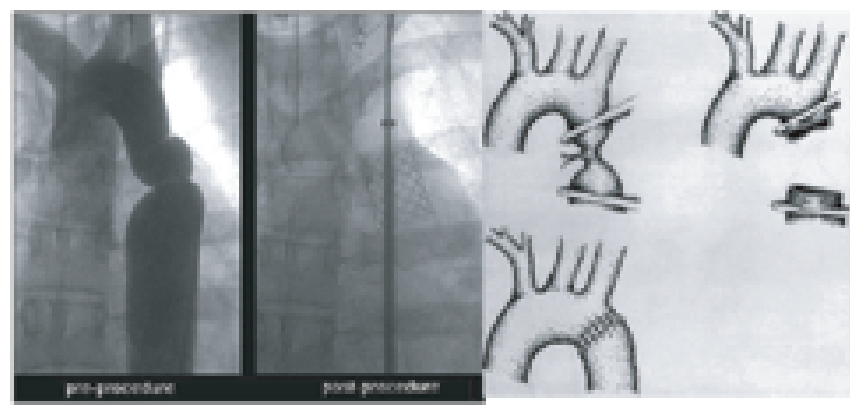

Figure 7: Coarctation Stenting

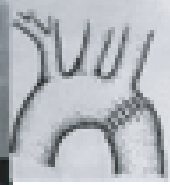

Figure 8: Surgical repair
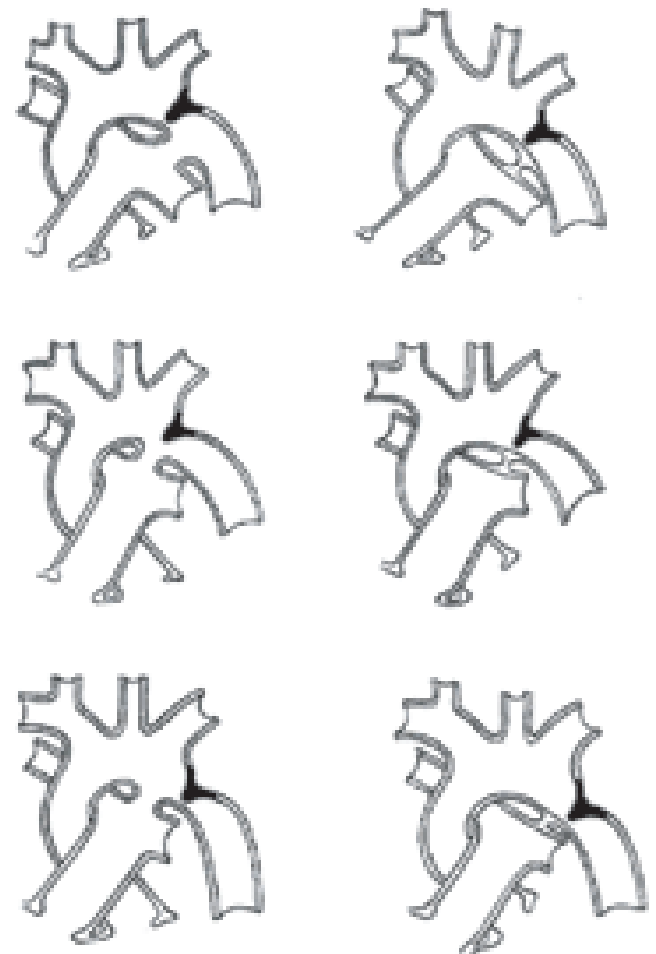

Figure 9: Varying relationships between coarctation of the aorta and the ductus arteriosus

\section{CONCLUSION}

Coarctation of the aorta is not simply a mechanical obstruction of the aorta. Life expectancy remains reduced in patients with coarctation of the aorta even after successful repair. Late arterial hypertension, more often systolic, which occurs in nearly one third of patients after repair, and atherosclerosis are the main determinants of cardiovascular events.

Coarctation of the aorta is a condition that requires life-long follow-up in a centre with expertise in this field and adequate invasive and non-invasive imaging facility. With the advent of readily available magnetic resonance imaging, balloon angioplasty, stenting and improved surgical techniques there is great opportunity for improvement in the long-term management of this condition.

\section{DISCLOSURE}

All the authors declared no competing interest. 


\section{REFERENCES}

1. Paediatric Cardiology. $2^{\text {nd }}$ edition. Robert H Anderson,Edward J Baker,,R F J Macartney,M L Rigby,E A Shinebourne,M Tynan.

2. Moss and Adams' Heart Disease in Infants, Children, and Adolescents: Including the Fetus and Young Adults, 7th Edition, Allen, Hugh D.; Driscoll, David J.; Shaddy, Robert E.; Feltes, Timothy F.

3. The Science and Practice of Pediatric Cardiology. $2^{\text {nd }}$ Edition.Arthur Garson Jr,J Timothy Bricker,David J Fisher,Steven R. Neish.

4. Isner JM, Donaldson RF, Fulton D, et al. Cystic medical necrosis in coarctation of the aorta: A potential factor contributing to adverse consequences observed after percutaneous balloon angioplasty of coarctation sites. Circulation 1987; 75:689-695.

5. Vascular Dysfunction After Repair of Coarctation of the Aorta Impact of Early Surgery Marcello de Divitiis, Carlo Pilla, Mia Kattenhorn, Mariutzka Zadinello, Ann Donald Circulation 2001; 104;I-165-I-170

6. Arterial Reactivity Is Significantly Impaired in Normotensive Young Adults After Successful Repair of Aortic Coarctation in ChildhoodHelena M. Gardiner; David S. Celermajer,Keld E. Sorensen; Dimitri Georgakopoulos, Jacqui Robinson, Odette Thomas, RN; John E. Deanfield, Circulation 1994; 89; 1745-17

7. Anomalies Associated with Coaretation of Aorta Particular Reference to Infancy By ANTON E. BECKER, MIEs J. BECKERAND JESSE E. EDWARDS, M.D. Circulation 1970; 41; 1067-1075

8. Long-Term Outcome (Up to 15 Years) of Balloon Angioplasty of Discrete Native Coarctation of the Aorta in Adolescents and Adults Mohamed Eid Fawzy, Mahmoud Awad, Walid Hassan, Yusuf Al Kadhi, Mohamed Shoukri, Fadel Fadley, J. Am. Coll. Cardiol. 2004; 43;1062-1067

9. Stent treatment for coarctation of the aorta:intermediate term follow up and technical considerationsB D Thanopoulos, L Hadjinikolaou, G N Konstadopoulou, G S Tsaousis, F Triposkiadis,P Spirou Heart 2000;84;65-70

10. The long-term management of the patient with an aortic coarctation repair L. Swan, N. Wilson, A. B. Houston, W. Doig, J. C. S Pollock and W. Stewart Hillis. European Heart Journal. 1998; 19: 382-386. 